\title{
Caractérisation des microorganismes thermotrophes impliqués dans la solubilisation du Phosphate Naturel Marocain au cours de la biotransformation anaérobie du gazon
}

\author{
Moussa S. HASSIMI ${ }^{1,2 *}$, Amal BOUKESKASS ${ }^{2,5}$, Issa ADAMOU ${ }^{3}$, \\ Zoubeirou A. MAYAKI ${ }^{4}$, Yedir OUHDOUCH ${ }^{5}$ et Mohamed HAFIDI ${ }^{2}$ \\ ${ }^{1}$ Département Environnement et Adaptations au Changement Climatique, Université de Tillabéri BP 175, \\ Tillabéri, Niger. \\ ${ }^{2}$ Laboratoire d'Ecologie et Environnement(L2E) (Unité Associée au CNRST, URAC32, Unité associée au \\ NRS), Faculté de Sciences Semlalia, Université Cadi Ayyad, Marrakech Morocco. \\ ${ }^{3}$ Département Production durable des Cultures, Université de Tillabéri BP 175, Tillabéri, Niger. \\ ${ }^{4}$ Département Biologie, Université Abdou Moumouni de Niamey, BP 10960, Niamey. \\ ${ }^{5}$ Laboratoire de Biologie et de Biotechnologie des Microorganismes, Faculté de Sciences Semlalia, Université \\ adi Ayyad, Marrakech Morocco. \\ *Auteur correspondant, E-mail : atpscontact@gmail.com, Tél : (00227) 97377838.
}

\section{RESUME}

L'utilisation de bactéries solubilisatrices du Phosphate Naturel a largement été étudiée au cours de ces dernières années. Cependant, la plupart des essais de bio-solubilisation sont conduits dans des conditions aérobies. La présente étude décrit la démarche d'isolement et de caractérisation morphologique et biochimique de 10 isolats sélectionnés à partir des bioréacteurs de fermentation anaérobie. L'objectif était d'isoler les bactéries anaérobies thermotrophes des réacteurs capables de solubiliser la roche phosphatière. L'isolement de souches nommées PSAB (Phosphate Solubilizing Anaerobic Bacteria) a été réalisé à partir de jus de fermentation anaérobie d'un mélange de gazon et du Phosphate Naturel issue de la mine de Youssoufia au Maroc $\left(\mathrm{PN}^{\mathrm{Y}}\right)$. L'aptitude de ces isolats à solubiliser le $\mathrm{PN}^{\mathrm{Y}}$ a été conduite sur les milieux de culture Pivovskaya (PVK), Yeast-Extract Dextrose, National Biotechnologies Research Institut of Phosphate et Milieu Minimum Synthétique (MMS). L'étude des caractères morphologique et physiologiques a conclu que tous les isolats actifs pour la solubilisation du $\mathrm{PN}^{\mathrm{Y}}$ appartiennent au genre Bacillus. Cette bactérie est capable de dissoudre le phosphate naturel et le phosphate tricalcique sur milieu PVK solide par apparition des halos transparents indiquant la dégradation de $\mathrm{P}$ insoluble incorporé au milieu comme seule source de phosphate. La caractérisation biochimique de la souche a permis son identification comme étant Bacillus subtilis.

(C) 2017 International Formulae Group. All rights reserved.

Mots clés : Microorganismes solubilisateurs de phosphate, fermentation anaérobie, Bacillus subtilis, Phosphate Naturel, gazon.

\section{Characterization of thermophilic phosphate solubilizing microorganisms during grassland anaerobic biotransformation}

\begin{abstract}
The use of Rock Phospahte solubilizing bacteria has been widely studied in recent years. However, most of the bio-solubilization assays are conducted under aerobic conditions. The objective was to isolate the
\end{abstract}


thermotrophic anaerobic bacteria from the reactors and to prove the ability of solubilizing rock phosphate during anaerobic fermentation. The present study describes the isolation, morphological and biochemical identification and molecular characterization of 10 isolates selected from anaerobic fermentation bioreactors. Isolation of strains called PSAB (Phosphate Solubilizing Anaerobic Bacteria) was carried out using anaerobic fermentation juice from a mixture of grassland and Rock Phosphate from the Youssoufia mine in Morocco. The ability of these isolates to solubilize the Rock Phosphate was carried out on the Pivovskaya media (PVK), Yeast-Extract Dextrose media, National Biotechnologies Research Institut's of Phosphate growth medium and Minimum Synthetic media (MS). The study of morphological and physiological characteristics showed that all isolates with $\mathrm{PN}^{\mathrm{Y}}$ solubilization action belong to the genus Bacillus. This bacterium has both tricalcium phosphate and rock phosphate dissolving activity on solid PVK medium through the appearance of the transparent halos indicating the degradation of insoluble $\mathrm{P}$ incorporated in the medium as unique source of phosphate. The biochemical characterization of the strain allowed it to be identified as Bacillus subtilis.

(C) 2017 International Formulae Group. All rights reserved.

Keywords: Phosphate Solubilizing Microorganisms, anaerobic fermentation, Bacillus subtilis, rock phosphate, grassland.

\section{INTRODUCTION}

Les microorganismes solubilisateurs de phosphate naturel (PSM) portent le même phénotype par l'observation d'un halo ou zone claire produite sur plaque de gélose autour de la colonie bactérienne (Zaidi et al., 2009) en raison de la production d'acides organiques dans le milieu environnant. La taille du halo diffère d'une espèce à l'autre. Ces PSM sont généralement isolés des sols rhizosphérique et non rhizosphériques, du rhizoplan, de la phyllosphère, des gisements de $\mathrm{PN}$ et même sur des sols stressés (Aarab, 2013). De nos jours, deux principaux milieux d'isolement standard des PSM existent. Il s'agit des milieux PVK (Pivovskaya) et NBRIP (National Biotechnologies Research Institut of Phosphate) respectivement mis au point par Pivovskaya (1948) et Nautiyal (1999). Ces milieux de criblage sélectionnent des microorganismes capables d'utiliser les formes insolubles du phosphate inorganique et de produire des zones claires autour des colonies. Ce qui témoigne de la production des acides organiques pouvant dissoudre le phosphate de calcium présent comme seule source de phosphore dans le milieu de culture.

L'isolement de nouvelles souches de microorganismes cellulolytiques capables de solubiliser du Phosphate Naturel (PN) ou roche phosphatière en utilisant du gazon comme substrat en anaérobiose pourrait être d'une grande utilité pour une agriculture efficiente. Le concept de l'ajout de microorganismes solubilisateurs de phosphate sur la roche phosphatière comme source de $\mathrm{P}$ soluble pour les cultures est une alternative économique et écologique pour l'agriculture moderne (Chang and Yang, 2009). Il s'agit d'une nouvelle approche de production de biofertilisant respectueux de l'environnement. Les microorganismes solubilisateurs de phosphate transforment le $\mathrm{P}$ insolubles en formes solubles par acidification, chélation, réactions d'échange et formation de polymères (Delvasto et al., 2006).

Par ailleurs, le développement des techniques moléculaires pour le diagnostic clinique, l'étude de la biodiversité bactérienne dans l'environnement et pour les programmes de recherche en biotechnologie ont permis la découverte de plusieurs espèces microbiennes notamment les bactéries (Liu et al., 2000; Nguimbi et al., 2003 ; Kandjimi et al., 2015). Parmi les techniques de caractérisation moléculaire, l'amplification du gène de séquences l'ARNr 16S par PCR est la technique la plus utilisée. Elle permet la détection sélective aussi bien des bactéries cultivables que celles non cultivables. L'emploi de ces méthodes modernes est courant dans l'identification de bactéries solubilisatrices du phosphate naturel (Pradhan et Sukla, 2005 ; Popavath et al., 2008; Khan et al., 2009 ; Jayadi et Ibrahim, 2013 ; Fankem et al., 2015 ; Midekssa et al., 2016). 
A notre connaissance, très peu d'études ont été rapportées sur les microorganismes thermotrophes cellulolytiques solubilisateurs du PN en conditions anaérobie. De telles conditions se rencontrent notamment dans les sols engorgés voire submergés, dans les rizières, les tourbières, et certaines zones de bas-fonds forestiers. Nos travaux précédents ont mis en évidence l'existence d'une solubilisation microbienne du PN en conditions anaérobie thermotrophe (Hassimi et al., 2013) au cours de la fermentation de substrat ligno-cellulosique.

Ainsi, l'objectif de ce travail est, d'une part, l'isolement, la purification de microorganismes thermotrophes impliqués dans la dégradation du gazon et la solubilisation du PN de Youssoufia $\left(\mathrm{PN}^{\mathrm{Y}}\right)$ en anaériobiose, et leur caractérisation biochimique d'autre part.

\section{MATERIEL ET METHODES}

\section{Matériel}

\section{Échantillons du gazon}

Les échantillons du gazon étudiés appartiennent au spécimen des vouchers de la collection de l'herbier Mark-UCAM de la Faculté des Sciences Semlalia sous le numéro 8242, le choix du substrat de fermentation est basé sur son abondance, en tant que déchets verts collectés sur la pelouse de l'université.

\section{Le Phosphate naturel PN}

Les échantillons du $\mathrm{PN}^{\mathrm{Y}}$ utilisés ont été collectés de la région de Youssoufia (Maroc) sur le site d'extraction à ciel ouvert du l'Office Chérifien de Phosphate (OCP). La composition minéralogique des échantillons est donnée par Ouardi (2008). Les échantillons ont été mis dans des flacons en plastic stériles et conservés à $4{ }^{\circ} \mathrm{C}$ avant utilisation.

\section{Condition de fermentation et préparation des solutions d'ensemencement}

Le jus de fermentation est obtenu à partir de mélange de gazon, d'échantillon de $\mathrm{PN}^{\mathrm{Y}}$ et de l'eau de robinet comme décrit dans nos travaux précédents (Hassimi et al., 2013).
Les solutions mères ont été préparées à partir du gazon, du $\mathrm{PN}^{\mathrm{Y}}$, de l'eau de robinet et des prélèvements issus des bioréacteurs aux stades (jours) : $\mathrm{T}_{0}, \mathrm{~T}_{20}, \mathrm{~T}_{60}$ et $\mathrm{T}_{90}$. Deux grammes de chaque échantillon (Gazon et $\mathrm{PN}^{\mathrm{Y}}$ ) sont suspendus dans $18 \mathrm{ml}$ de solution saline stérile à $0,9 \%$ (poids/volume). Les solutions ont été ensuite agitées à l'aide d'un vortex en présence de billes de verre stériles $(3 \times 5$ minutes), puis traitées par les ultrasons pendant $10 \mathrm{~min}$ dans un bain de glace (Ouhdouch, 1996). $1 \mathrm{ml}$ de chaque solution obtenue a été dilué de $10^{-1}$ à $10^{-7}$.

\section{$L$ 'isolement des PSM}

L'isolement des PSM a été réalisé sur plusieurs milieux de cultures. Les solutions ont été ensuite traitées comme décrit précédemment. La sélection des microorganismes PSM, capables de solubiliser le tricalcique (TCP) et /ou la roche phosphatière (RP), en anaérobie, a été basée sur la capacité à utiliser TCP ou RP comme seule source de phosphore. Elle a été conduite sur la base de leur fonction solubilisatrice à travers des milieux de cultures référencés. Il s'agit des milieux PVK (Pikovskaya, 1948), NBRIP (Nautyal, 1999), MMS (Hamdali et al., 2008) et YED (Extraits de Levures). Des aliquotes de $100 \mu \mathrm{l}$ suspension sont déposées sur le milieu PVK. Les boîtes ensemencées sont incubées en anaérobiose à $45{ }^{\circ} \mathrm{C} \pm 2$ pendant 7 jours. Nous avons mesuré pour chaque isolat le diamètre total (diamètre halo + diamètre colonie), et le diamètre de colonie. Le diamètre du halo de solubilisation a été calculé en soustrayant le diamètre de colonie du diamètre total.

\section{Etude morphologique au microscope optique et électronique à balayage (MEB) \\ Culture sur lamelle}

L'étude morphologique des isolats et souches sélectionnés est menée au microscope optique à différents grossissements et au microscope électronique à balayage (MEB). Pour cela, une culture de la souche isolée a été réalisée sur lamelle (Figure 1). Les observations ont été faites à partir des 
différents milieux servant à l'étude des spores (position, ajustement et la forme des spores).

\section{Culture sur lame}

Quelques millilitres de milieu nutritif gélosé en surfusion sont répartis à la surface d'une lame stérile posée sur un support dans une boîte de Pétri. Après refroidissement et prise en masse de cette gélose, quelques gouttes du même milieu gélosé servent à former deux rails surélevés, disposé dans le sens de la largeur de la lame. Une goutte d'inoculum est placée entre les deux rails de façon à ce que l'inoculum entre en contact avec elle (Figure 2). Avant de placer l'ensemble à l'étuve d'incubation, quelques millilitres d'eau stérile sont versés au fond de la boîte de Pétri afin d'éviter la dessiccation rapide du milieu gélosé. Après 14 jours d'incubation à $28{ }^{\circ} \mathrm{C}$, les spores et les structures cellulaires sont examinés au microscope optique, Ouhdouch, 1996.

Etude de l'activité antimicrobienne sur milieux solides

\section{Tests de confrontation}

Les souches purifiées et identifiées, PSM, ont été ensemencées en strie rectiligne qui partage la boîte de pétri en deux parties égales. Après incubation à $28{ }^{\circ} \mathrm{C}$ pendant deux semaines, on ensemence les souches indicatrices perpendiculairement à la strie formée par la croissance de l'isolat investigué. La lecture du résultat d'inhibition est faite après incubation durant un temps et une température favorables aux souches indicatrices.

\section{Test de cylindre d'agar}

L'activité antibactérienne et l'activité antifongique sont déterminées par la technique des cylindres d'agar. Cette technique consiste à ensemencer en stries serrées les souches PSAB sur boîtes de Pétri contenant un milieu de production (gélose nutritive). Après incubation des cultures à $45{ }^{\circ} \mathrm{C}$ pendant une semaine, trois cylindres de gélose de chaque culture $(6 \mathrm{~mm}$ de diamètre) sont prélevés à l'aide d'un emporte-pièce et déposés soit sur de la gélose nutritive pour les bactéries soit, sur le milieu Sabouraud pour les levures et les moisissures. Les boîtes sont ensemencées en surcouche avec les micro-organismes tests présentés dans le tableau 10 , incubées à $30^{\circ} \mathrm{C}$ pendant $48 \mathrm{~h}$ pour les bactéries et $72 \mathrm{~h}$ pour les moisissures. Un témoin formé avec un cylindre de la gélose sans culture de PSAB est mis dans chaque boîte de Pétri. Les boîtes sont laissées $2 \mathrm{~h}$ à $+4{ }^{\circ} \mathrm{C}$ pour permettre une diffusion des produits actifs. Les zones d'inhibition apparaissent autour des cylindres d'agar après $24 \mathrm{~h}$ d'incubation à $28^{\circ} \mathrm{C}$.

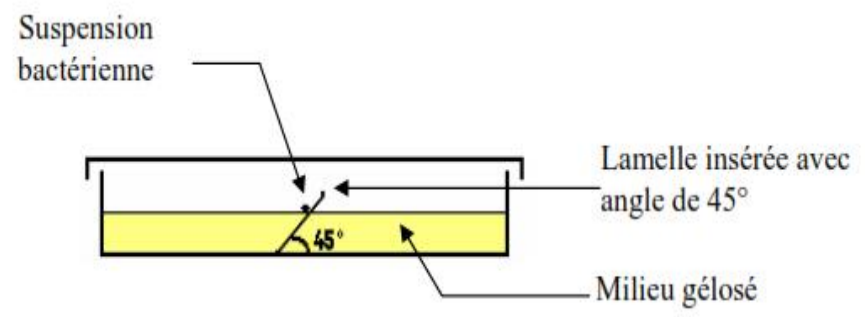

Figure 1: Schéma présentant la culture sur lamelle. 


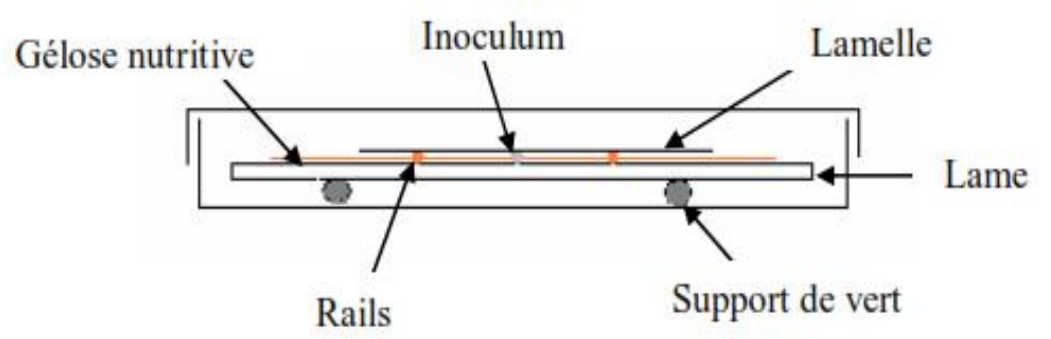

Figure 2: Schéma présentant la culture sur lame.

\section{RESULTATS}

\section{Isolement et sélection des PSAM}

La première étape de l'isolement des microorganismes impliqués dans la transformation du gazon et la solubilisation de la roche phosphatière en anaérobiose a été réalisée sur le milieu PVK à partir des constituants nutritifs du bioréacteur. Les résultats sont consignés dans les Tableaux 1 et 2. Dans nos conditions expérimentales, nous avons noté la présence uniquement de bactéries avec colonies de mophologie caractéristiques du genre Bacillus. Aucune colonie caractéristique de champignon n'a été observée malgré leur capacité cellulolytique et solubilisatrice du PN connue (Nguimbi et al., 2003).

Nous avons noté l'absence des actinobactéries sur tous les milieux y compris le MMS. Ces résultats sont contraires à ceux qui ont montré la présence de nouvelle souche d'actinobactéries sur la roche phosphatière de Youssoufia. Nous avons également été surpris par l'absence de diversité morphologique des colonies sélectionnées sur les différents milieux testés. Les bactéries dissolvant le phosphate tricalcique, ou les PSM, s'entourent sur milieu PVK solide par des halos transparents indiquant la dégradation de $\mathrm{P}$ tricalcique insoluble incorporé au milieu comme seule source de phosphore (Figure 3).

L'absence des PSM lors de l'analyse du RP de Youssoufia est inattendue, ce qui explique l'absence de microorganismes dans la roche. Les résultats du screening des PSM par les autres milieux d'isolements n'a pas permis d'améliorer la diversité morphologique des colonies Tableau 2. Vu que le diamètre des halos de solubilisation est proportionnel à l'efficience des bactéries pour la solubilisation du $\mathrm{P}$ inorganique, on s'est basé sur le diamètre des halos qui doit être au moins égal à $0,4 \mathrm{~cm}$, pour le criblage des isolats les plus performants afin de poursuivre d'autres tests. Ainsi, le nombre d'isolat solubilisant efficacement le $\mathrm{P}$ tricalcique a été réduit. Une dizaine a été testée pour leur pouvoir solubilisateur de $\mathrm{P}$ inorganique. Le Tableau 3 récapitule les différents résultats obtenus concernant les premiers critères du criblage. Ainsi, la solubilisation du $\mathrm{P}$ inorganique par les microorganismes est influencée par la présence de $\mathrm{P}$ soluble dans le milieu.

\section{Solubilisation des autres sources de $P$ inorganique \\ La sélection des PSAB se fait} classiquement dans des milieux de culture renfermant le $\mathrm{P}$ calcique comme seule source de P. Or, dans les sols acides, le P soluble est rapidement précipité par les cations du sol, $\mathrm{Fe}^{3+}$ et $\mathrm{Al}^{3+}$ en donnant des complexes insolubles. De ce fait, on a remplacé le $\mathrm{P}$ tricalcique par d'autres sources de $\mathrm{P}$ inorganique communément présentes dans les deux types du sol, acide et alcalin. Cette expérience a été menée par la méthode de colonisation. Les suspensions des 10 isolats 
purifiés sont ensemencées par inondation sur les milieux PVK, NBRIP et YED sans aucune source du phosphore. Les poudres de diverses sources de $\mathrm{P}$ inorganiques stériles sont apportées par spot (point de dépôt). Dans ce test, la croissance est contrôlée par la capacité à coloniser le spot.

Les résultats obtenus montrent que la majorité des isolats évalués colonisent les points de dépôt et s'entouraient des petits halos de solubilisation dans les boîtes renfermant des autres sources de $P$. L'importance des diamètres des halos diffèrent d'un milieu à l'autre pour la même bactérie. Les halos ont été plus clairs sur le milieu PVK contenant $\mathrm{CaHPO}_{4}$ comme seule source de $\mathrm{P}$ insoluble (Figure 3 ). Les résultats présentés dans le Tableau 4 montrent que les isolats sont capables de solubiliser les autres sources de phosphore minéral qui ont substitué le $\mathrm{P}$ tricalcique dans le milieu PVK. En effet, des halos de solubilisation ont été observés autour des 10 isolats dans les boîtes renfermant $\mathrm{Ca}_{5} \mathrm{HO}_{13} \mathrm{P}_{3}$ et $\mathrm{CaHPO}_{4}$ comme source de $\mathrm{P}$, tandis qu'elles sont toutes incapables de dissoudre le $\mathrm{P}$ du fer et d'aluminium.

Caractérisation taxonomique du Bacille morphotype sélectionné (ECOLAB)

Caractéristiques culturales des souches solubilisatrices du $P N^{Y}$

Les caractéristiques morphologiques et culturales de la souche PSAB ont été étudiées. Les différentes observations concernant la croissance, le développement et la pigmentation des colonies, la présence des spores en anaérobiose et en aérobiose ainsi que la présence de pigments diffus dans la gélose, après incubation de 3 semaines, sont groupés dans le Tableau 5. L'isolat se développe sur tous les milieux préconisés pour les bactéries solubilisatrices du phosphate ainsi que sur la gélose nutritive.

Ces résultats confirment l'étude morphologique de souche au microscope optique et au microscope électronique à balayage (Figure 4).

Caractères microscopiques

L'analyse des résultats de la caractérisation microscopique et Gram a révélé qu'il s'agit de bactérie de type Bacille Gram positif, anaérobie facultatif, formant des spores en aérobiose (Figures 4).

\section{Caractères physiologiques \\ Utilisation de substrats carbonés}

Pour l'étude physiologique de l'isolat sélectionné, les critères d'analyse pour les espèces du genre Bacillus les plus proches ont été utilisés notamment la production de pigment, l'utilisation de différentes sources de carbones. Les résultats de l'utilisation des différentes sources de carbones sont réunis dans le Tableau 6. La souche assimile tous les substrats carbonés dans les conditions aérobies contre cinq substrats carbonés en anaérobiose (citrate de sodium, glucose, Dgalactose, lactose et faiblement le raffinose).

L'analyse des caractères morphologiques et physiologiques de l'isolat sélectionné et la compilation des mêmes données avec les espèces du genre Bacillus les plus étudiés dans la littérature, nous renseigne un peu plus sur l'identité probable de la souche. D'après notre étude, la souche sélectionnée présente les mêmes caractères que le Bacillus subtilis (Tableau 7).

\section{Spectre d'activité des trois souches actives}

En utilisant la technique des cylindres d'agar, le spectre d'activité antimicrobienne de la souche sélectionnée sur trois milieux de production a été déterminé vis-à-vis de plusieurs micro-organismes tests. Les résultats sont consignés dans le Tableau 8 et montrent que la souche est active contre E.coli, M.luteus, B.subtilis et B.cereus. La souche présente une activité contre le M. luteus sur les trois milieux de production et vis-à-vis du B. cereus sur les deux milieux gélose nutritive et Bennett. 
Tableau 1 : Isolement des PSAM à partir des constituants du mélange réactionnel.

\begin{tabular}{llll}
\hline Origine d'isolat & Gazon & PN $^{\mathrm{Y}^{*}}$ & Eau $^{{ }^{* *}}$ \\
\hline Nombre d'UFC $\left(\mathbf{x ~ 1 0}^{3}\right)$ sur PVK & 35 & 0 & 0
\end{tabular}

$\left(*=\mathrm{PN}^{\Upsilon}\right)$, la roche phosphatière de Youssoufia ; $(* *)$, eau du robinet utilisée pour préparer le jus de fermentation dans les bioréacteurs.
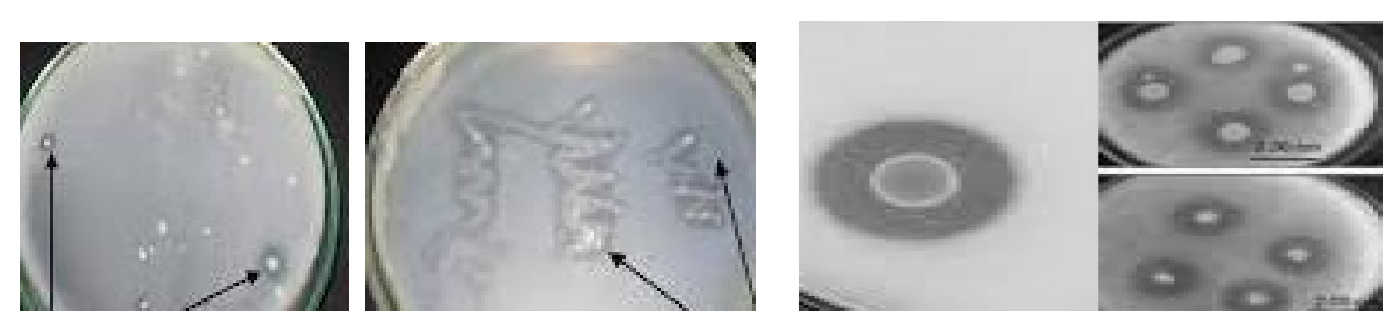

Figure 3 : Test qualitatif de reconnaissances des microorganismes solubilisateurs de $\mathrm{P}$ tricalcique sur PVK solide montrant les halos de solubilisation.

Tableau 2: Isolement des PSAM au cours de la transformation du gazon en anaérobiose sur différents milieux.

\begin{tabular}{|c|c|c|c|c|c|}
\hline Stades d'incubation (jours) & $\mathbf{0}$ & 20 & 30 & 60 & 90 \\
\hline Nombre d'UFC (x 10 $\left.1^{5}\right)$ sur PVK & 0 & 150 & 250 & 260 & 267 \\
\hline Nombre d'UFC (x 10 $\left.{ }^{5}\right)$ sur MMS & 0 & 120 & 130 & 127 & 135 \\
\hline Nombre d'UFC (x 10 $\left.{ }^{5}\right)$ sur YED & 1 & 200 & 230 & 132 & 230 \\
\hline Nombre d'UFC (x 10 $\left.1^{5}\right)$ sur NBRIP & 0 & 210 & 240 & 256 & 260 \\
\hline
\end{tabular}

Tableau 3 : Diamètre des halos de solubilisation de $\mathrm{P}$ et la coloration Gram des isolats testés sur le PVK.

\begin{tabular}{lll}
\hline Isolats & Halos $(\mathbf{c m})$ & Gram \\
\hline G1 & $0,9( \pm 0,2)$ & + \\
\hline G2 & $0,8( \pm 0,1)$ & + \\
\hline G3 & $0,7( \pm 0,1)$ & + \\
\hline G4 & $0,8( \pm 0,15)$ & + \\
\hline G5 & $0,7( \pm 0,12)$ & + \\
\hline G6 & $1,1( \pm 0,12)$ & + \\
\hline G7 & $0,9( \pm 0,15)$ & + \\
\hline G8 & $0,7( \pm 0,2)$ & + \\
\hline G9 & $1,2( \pm 0,15)$ & + \\
\hline G10 & $1( \pm 0,15)$ & + \\
\hline
\end{tabular}


Tableau 4 : Solubilisation des autres sources de $\mathrm{P}$ inorganique et évaluation des autres milieux de base utilisés pour la sélection des bactéries solubilisatrices du P (NBRIP, YED-P).

\begin{tabular}{|c|c|c|c|c|c|c|}
\hline \multirow[t]{2}{*}{ Isolats } & \multicolumn{4}{|c|}{ PVK modifié } & \multirow[t]{2}{*}{ NBRIP } & \multirow[t]{2}{*}{ YED-P } \\
\hline & $\mathrm{CaHPO}_{4}$ & $\mathrm{Ca}_{5} \mathrm{HO}_{13} \mathrm{P}_{3}$ & $\mathrm{FePO}_{4}$ & $\mathrm{AlPO}_{4}$ & & \\
\hline G1 & + & + & - & - & + & + \\
\hline G2 & + & + & - & - & + & + \\
\hline G3 & + & + & - & - & + & + \\
\hline G4 & + & + & - & - & + & + \\
\hline G5 & + & + & - & - & + & - \\
\hline G6 & + & + & - & - & + & + \\
\hline G7 & + & + & - & - & + & + \\
\hline G8 & + & + & - & - & + & - \\
\hline G9 & + & + & - & - & + & + \\
\hline G10 & + & + & - & - & + & + \\
\hline
\end{tabular}

$(+) /(-)$, présence/absence de halo autour des colonies

Tableau 5 : Caractéristiques culturales de l'isolat sélectionné.

\begin{tabular}{lcc}
\hline & Anaérobiose & Aérobiose \\
\hline NBRP & Crème & Blanc \\
\hline PVK & Crème & Blanc \\
\hline MMS & Crème & Blanc \\
\hline Gélose nutritive (GN) & Blanc & Blanc \\
\hline
\end{tabular}

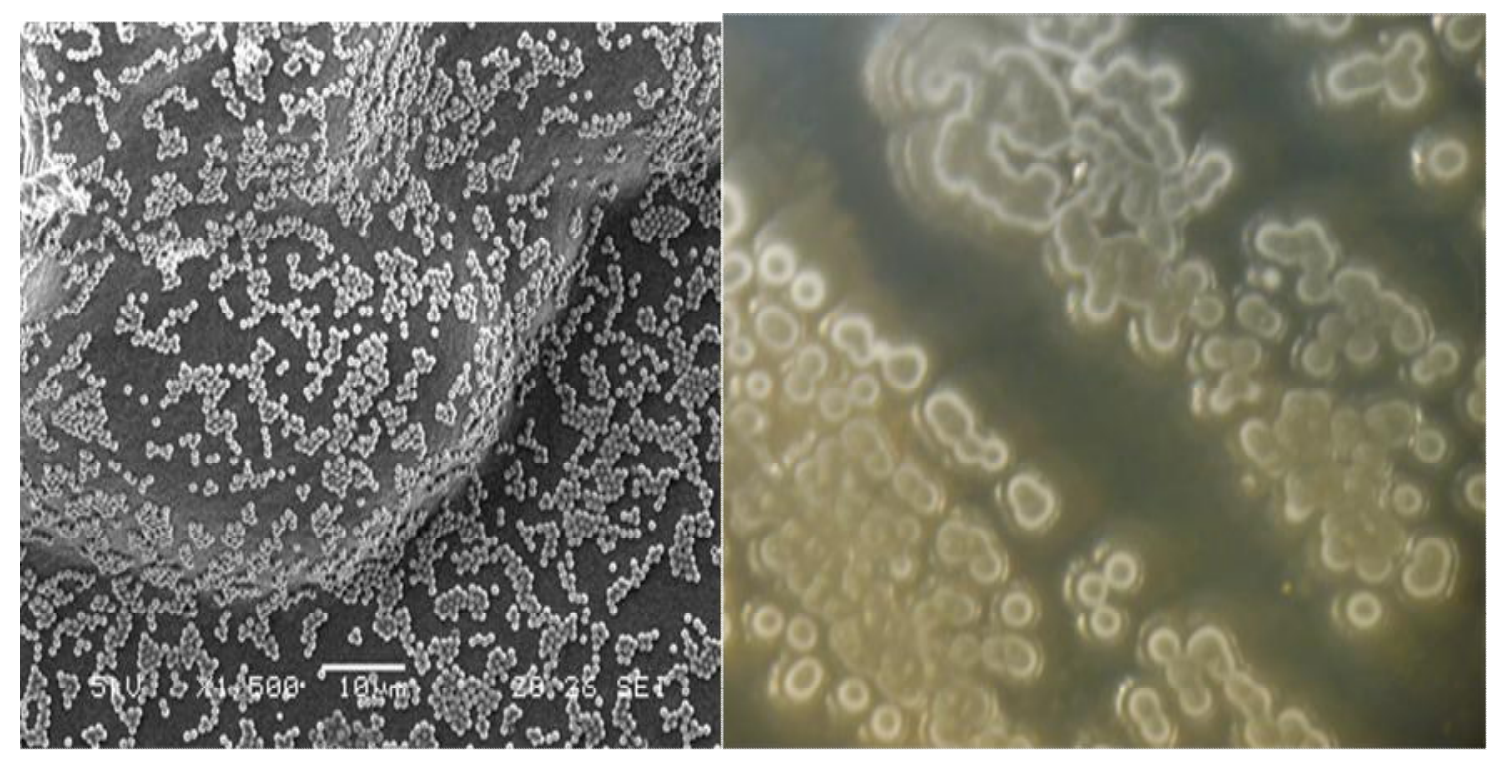

Figure 4 : Image microscopie optique et électronique à balayage d'isolats de Bacillus sur milieu gélosé. 
Tableau 6 : Métabolisme glucidique de la souche sélectionnée.

\begin{tabular}{lcc}
\hline Substrats carbonés & Aérobiose & Anaérobiose \\
\hline Sorbitol & $+/-$ & - \\
\hline Sodium citrate & + & + \\
\hline D-Xylose & + & - \\
\hline Raffinose & $+/-$ & $+/-$ \\
\hline Maltose & + & - \\
\hline D-Fructose & + & - \\
\hline Mannitol & + & - \\
\hline Glucose & + & + \\
\hline L-Mannose & + & - \\
\hline Sucrose & $+/-$ & - \\
\hline L-Arabinose & + & - \\
\hline D-Galactose & + & + \\
\hline Dextrine & + & - \\
\hline Lactose & + & + \\
\hline Inositol & + & - \\
\hline Rhamnose & + & - \\
\hline$\quad+:$ développement de la souche (Substrat utilisé). & \\
$\quad-:$ absence de développement de la souche (Substrat non utilisé). & \\
$\quad+-$ : développement modéré de la souche (Substrat faiblement utilisé). &
\end{tabular}

Tableau 7: Caractéristiques phénotypiques et phylogénétiques distinguant Bacillus substilis ECOLAB et les autres espèces de Bacillus.

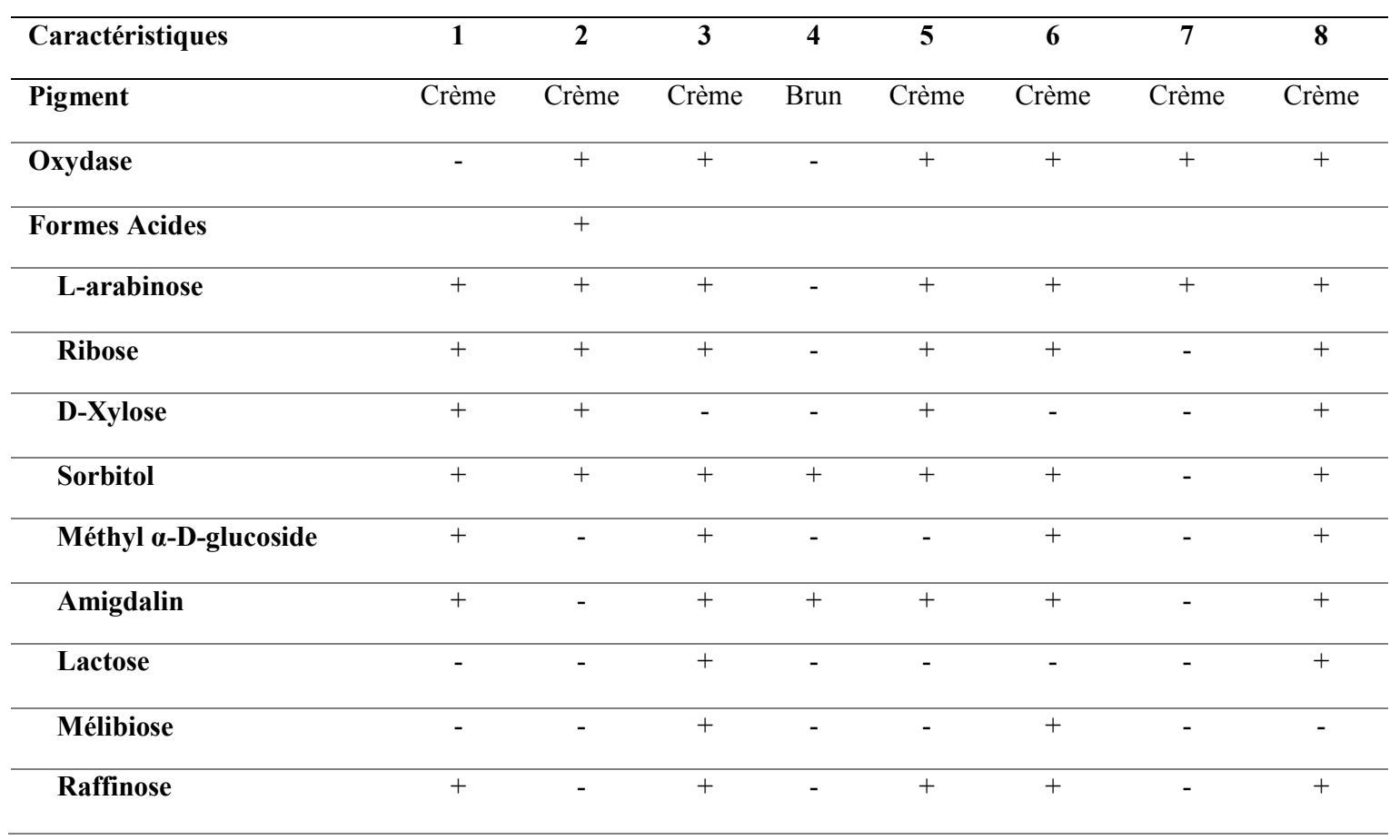




\begin{tabular}{|c|c|c|c|c|c|c|c|c|}
\hline Trehalose & + & + & + & - & + & + & + & + \\
\hline Amidon & + & + & + & - & + & + & - & + \\
\hline Glycogène & + & + & - & - & - & - & - & + \\
\hline Gentibiose & + & - & - & - & - & - & - & - \\
\hline D-Turanose & - & - & - & - & - & + & - & - \\
\hline Utilisation du citrate & + & - & + & + & + & + & + & - \\
\hline $\begin{array}{l}\text { Production de } \\
\text { Dihydroxyacétone }\end{array}$ & + & - & - & - & - & - & - & + \\
\hline Hydrolyse de l'ONPG & + & + & - & + & + & + & + & + \\
\hline $\begin{array}{l}\text { Croissance avec lysozymes } \\
(0.001 \%)\end{array}$ & + & + & - & - & - & - & - & - \\
\hline Lecithinase & + & + & - & + & + & - & + & - \\
\hline Arginine dihydrolase & + & - & - & - & - & + & - & - \\
\hline Lysine décarboxylase & + & - & - & - & - & - & - & - \\
\hline Ornithine décarboxylase & + & - & - & - & - & - & - & - \\
\hline
\end{tabular}

\section{Sensibilité à :}

\section{Ceftadizine (30 $\mu \mathrm{g})$}

Colistin (10 $\mu \mathrm{g})$

$\begin{array}{ccccccccc}\text { Souches }: \mathbf{1}, B \text {, axarquiensis } \mathrm{CR}-119^{\mathrm{T}} ; \mathbf{2}, & \text { B. malacitensis } & \mathrm{CR}-95^{\mathrm{T}} ; \mathbf{3}, & \text { B. amyloliquefaciens } & \text { DSM } 7^{\mathrm{T}} ; \mathbf{4}, \text { B. atrophaeus } \text { DSM }\end{array}$ $7264^{\mathrm{T}}$; 5, B. mojavensis DSM $9205^{\mathrm{T}}$; 6, B. subtilis ECOLAB ; 7, B. Vallismortis DSM $11031^{\mathrm{T}} ; \mathbf{8}$, B. velezensis CECT $5686^{\mathrm{T}}$. Résultats comparé aux travaux de Claus et Berkley (1986), Logan et Berkley (1984), Nakamura (1989), Roberts et al. (1994) et Ruiz-Garcia et al. (2005).

Tableau 8 : Spectre d'activité de la souche PSAB.

\begin{tabular}{|c|c|c|c|}
\hline \multirow[t]{2}{*}{ Micro-organismes tests } & \multirow[t]{2}{*}{ Milieux de cultures } & \multicolumn{2}{|c|}{ Activité } \\
\hline & & Aérobiose & Anaérobiose \\
\hline \multirow[t]{2}{*}{ C. tropicalis } & GN & - & NT \\
\hline & $\mathrm{B}$ & - & NT \\
\hline \multirow[t]{2}{*}{ F. oxysprorum fsp. albedinis } & GN & - & NT \\
\hline & $\mathrm{B}$ & + & NT \\
\hline \multirow[t]{2}{*}{ E. coli } & GN & - & - \\
\hline & $\mathrm{B}$ & - & - \\
\hline \multirow[t]{3}{*}{ M. luteus } & GN & + & + \\
\hline & B & + & + \\
\hline & GBAm & + & + \\
\hline \multirow[t]{3}{*}{ B.subtilis } & $\mathrm{GN}$ & - & + \\
\hline & B & + & + \\
\hline & GBAm & - & - \\
\hline
\end{tabular}

GN: gélose nutritive; B: Bennett; GBAm: GBA modifié; NT: non testé. 


\section{DISCUSSION}

Dans cette étude, les isolats sélectionnés ont été caractérisés à l'aide de critères morphologiques, physiologiques et biochimiques. Les cellules sont des bactéries baciliformes, Gram-positives, crème pigmentée, aéro-anaérobie. Les tests biochimiques et les analyses comparatives avec d'autres souches proches (Tableaux 6 et 7) orientent l'identification de la souche vers l'espèce Bacillus subtilis. Ces résultats concordent avec les travaux précédents de Hassimi et al. (2013) qui a procédé à une caractérisation moléculaire de la souche isolée du bioréacteur de fermentation anaérobie dans les mêmes conditions. D'après les auteurs, l'identification moléculaire montre que cette souche présente un pourcentage de similarité supérieur à $98 \%$ avec la souche Bacillus subtilis. L'absence des PSM lors de l'analyse du RP de Youssoufia est inattendue. En effet, des travaux antérieurs ont montré le contraire (Hueur et al., 1997 ; Hamdali et al., 2008). Il est possible de proposer comme explication, les conditions expérimentales notamment l'anaérobiose couplée à la thermotrophie (Rivière, 1975 ; Stackebrandt et al., 1991 et Hassimi et al., 2013). En outre, il s'agit d'un minerai qui a subi un traitement par calcination (Khaddor et al., 1997; Ouardi, 2008), ce qui justifierait l'absence de microorganismes dans la roche. Les résultats du screening des PSM par les autres milieux d'isolements décrits dans la littérature (Aarab, 2013) n'a pas permis d'améliorer la diversité morphologique des colonies. Goldstein et al. (2003) ont montré que la solubilisation du P minéral est codée par un gène spécifique chez les microorganismes actifs sur le matériau phosphaté. Ces derniers ont démontré cette faculté génétique en transférant le cluster du gène codant pour la solubilisation $\mathrm{du} P$ minéral, sur une souche d'Echerichia coli non solubilisatrice ( $\left.\mathrm{PSM}^{-}\right)$. Cette souche génétiquement modifiée montre une activité de solubilisation vis-à-vis du $\mathrm{P}$ minéral. Cependant, l'expression $\mathrm{du}$ gène est influencée par la présence ou l'absence de source de P soluble dans le milieu. Cela suggère que le processus de solubilisation implique la production d'acides organiques (Hassimi et al., 2013). Toutes les souches sélectionnées ont solubilisé le TCP avec formation des halos sur le milieu Pikovskaya (Tableau 4). Cela suggère que tous les isolats ont pu produire de la phosphatase. Cette dernière n'agit pas directement sur la solubilisation inorganique de $\mathrm{P}$, mais l'activité de la phosphatase peut contribuer à abaisser le $\mathrm{pH}$ du milieu de culture par l'action déphosphorylatrice et la production d'acides (Achal et al., 2007). Notre étude a démontré que la capacité de la souche Bacillus subtilis à solubiliser le TCP est presque la même pour tous les isolats vu les diamètres des halos (Tableau 3). Parmi toutes les souches qui poussent sur les milieux de sélection standards (PVK, NBRIP), les rapports dans la littérature suggèrent que la solubilisation microbienne du phosphate minéral peut être due à l'excrétion d'acides organiques provoquant l'acidification $\mathrm{du}$ milieu externe ou à l'excrétion de substances chélatantes telles que les sidérophores (Hafidi, 1996 ; Whitelaw, 2000 ; Hassimi, 2014). L'acide carbonique et les acides organiques produites lors de la décomposition des matières organiques solubilisent le PN, ce qui entraine la libération de phosphate et de calcium dans la solution. En effet, au cours de la fermentation anaérobie, il y a une phase acidogène traduite par la production massive d'acides organiques issus de la biodégradation de la matière organique. C'est au cours de cette phase que le PN se solubilise essentiellement à cause de la baisse $\mathrm{du} \mathrm{pH}$. Bacillus subtilis est longtemps étudiée et est connue pour sa capacité à se développer dans des conditions anaérobies par voie de fermentation du substrat carboné présent dans le milieu et par utilisation du nitrate ou du nitrite comme accepteur final d'électron. Ainsi, nous concluons que cette souche est capable de solubiliser le $\mathrm{PN}^{\mathrm{Y}}$ au cours de la fermentation anaérobie thermotrophe de substrat lignocellulosique. À ce jour, seuls quelques travaux 
ont révélé l'existence de PSM thermotolérants aérobies isolés du compost et des biofertilisants (Chang et Yang, 2009 ; Xiao et al., 2011).

En comparant les caractères morphologiques culturaux et physiologiques, des deux souches (isolat sélectionné PSAB ECOLAB et Bacillus subtilis B. sType) on se rend compte que les activités biologiques entre les deux souches sont différentes de point de vue production de molécules antibactériennes, ce qui laisse penser qu'il s'agit d'une nouvelle souche de Bacillus subtilis. De plus, la confrontation entre les deux souches PSAB et Bacillus subtilis ${ }^{\mathrm{T}}$ sur le milieu de production Bennett (résultats non présentés), a montré que la souche PSAB ECOLAB produit des substances qui inhibent la croissance de la souche Bacillus subtilis. Ce qui confirme la différence entre les 2 souches de point de vue production de métabolites secondaires d'où la différence taxonomique probable des deux souches. L'hybridation DNA-DNA entre les deux souches, PSAB et Bacillus subtilis ${ }^{\mathrm{T}}$ est donc nécessaire pour compléter l'identification taxonomique des deux souches.

\section{Conclusion}

Cette étude a montré que la souche Bacillus subtilis isolée et caractérisée lors de la fermentation anaérobie thermotrophe de substrat lignocellulosique est capable de solubiliser la roche phosphatière $\mathrm{PN}^{\mathrm{Y}}$ et le TCP. Pour la première fois, à notre connaissance, cette bactérie porte le phénotype PSAB (Phosphate Solubilizing Anaerobic Bacteria). Une étude poussée telle que l'hybridation DNA-DNA entre les deux souches, PSAB et Bacillus subtilis ${ }^{\mathrm{T}}$ serait nécessaire pour confirmer qu'il s'agirait bien d'une nouvelle souche de Bacillus subtilis.

\section{CONFLIT D'INTERETS}

Les auteurs déclarent qu'il n'existe aucun conflit d'intérêts pour cet article.

\section{CONTRIBUTIONS DES AUTEURS}

MSH est l'instigateur du projet et $\mathrm{AB}$ a participé à la rédaction. Les travaux ont été réalisés dans le Laboratoire de Microbiologie sous la supervision de YO et le Laboratoire Ecologie \& Environnement sous la supervision de $\mathrm{MH}$. IA et AZM ont contribué dans la rédaction et la correction du manuscrit.

\section{REMERCIEMENTS}

Nous adressons nos remerciements à l'Agence Universitaire de la Francophonie pour son soutien financier pour la réalisation des travaux qui ont conduit à cet article.

\section{REFERENCES}

Aarab S. 2013. Sélection et caractérisation de bactéries solublisatrices de phosphates isolées à partir des sols rhizosphériques de riz et de légumineuses du nord marocain. Thèse Doctorat. Faculté des Sciences et Techniques Tanger, p.218

Achal V, Savant VV, Reddy MS. 2007. Phosphate solubilization by a wild type strain and UV-induced mutants of Aspergillus tubingensis. Soil Biol Biochem., 39: 695.

Chang CH, Yang SS. 2009. Thermo-tolerant phosphate-solubilizing microbes for multi-functional biofertilizer preparation. Bioresour. Technol., 100:1648-1658.

Claus D, Berkeley RCW. Genus Bacillus. 1986. In Bergey's Manual of Systematic Bacteriology, Sneath PHA, Mair NS, Sharpe ME, Holt JG (eds). Williams \& Wilkins: Baltimore, 2; 1105-1139.

Delvasto P, Valverde A, Ballester A, Igual JM, Muñoz JA, González F, Blázquez ML, García C. 2006. Characterization of brushite as a re-crystallization product formed during bacterial solubilization of hydroxyapatite in batch cultures. Soil Biol. Biochem., 38: 2645-2654.

Fankem H, Mafokoua HL, Ngo Nkot L, Simo C, Tchouomo DD, Tchuisseu TGV, Nwaga D, Etoa F-X. 2015. Biodiversity of the phosphate solubilizing 
microorganisms (PSMs) population from the rice rhizosphere soils of the two agro-ecological zones of Cameroon. Int. J. Biol. Chem. Sci., 9(5): 2284-2299. DOI: http://dx.doi.org/10.4314/ijbcs.v9i5.3

Goldstein A, Lester T, Brown J. 2003. Research on the metabolic engineering of the direct oxidation pathway for extraction of phosphate from ore has generated preliminary evidence for PQQ biosynthesis in Escherichia coli as well as a possible role for the highly conserved region of quinoprotein dehydrogenases. Biochimica et Biophysica Acta (BBA) - Proteins and Proteomics, 1647(1-2) : 266-271.

Hafidi M. 1996. Contributions à la valorisation des composts par additions de phosphates naturels marocains. Thèse Doctorat d'Etat FSS Marrakech, p.155.

Hamdali H, Hafidi, M, Virolle, M. J., Ouhdouch, Y. 2008. Rock phosphatesolubilizing Actinomycetes: screening for plant growth-promoting activities. World J Microbiol Biotechnol., 24: 2565-2575.

Hassimi SM, Hamdali H, Ouhdouch Y, Pinelli E, Merlina G, Revel JC, Hafidi M. 2013. Moroccan rock phosphate solubilization during a thermo-anaerobic grassland waste biodegradation process. African Journal of Biotechnology, 12(49) : 68596865. DOI: 10.5897/AJB2013.12804

Hassimi SM. 2014. Contribution à l'étude des mécanismes de la solubilisation du phosphate naturel au cours de la biotransformation anaérobie des déchets organiques. Thèse PhD, INP-Université Paul Sabatier de Toulouse, Toulouse. p176.

Hueur H, Krsek M, Baker P, Smalla K, Wellington EMH. 1997. Analysis of actinomycete communities by specific amplification of genes encoding $16 \mathrm{~S}$ rRNA and gel-electrophoretic separation in denaturing gradients. Appl. Environ. Microbiol., 63, 3233-3241.
Jayadi MB, Ibrahim B. 2013. In vitro selection of rock phosphate solubility by microorganism from Ultisols in South Sulawesi, Indonesia. American Journal of Agriculture and Forestry, 1(4): 68-73.

Kandjimi OS, Uzabakiriho J-D, Chimwamurombe PM. 2015. Isolation and characterization of culturable bacteria from bulk soil samples and the rhizosphere of arid-adapted Tylosema esculentum (Burchell). A. Schreiber (Marama bean) in Namibia. African Journal of Biotechnology, 14(11): 944952. DOI: 10.5897/AJB2014.14257

Khaddor M, Ziyad M, Halim M, Joffre J, Amblès A. 1997. Characterization of soluble organic matter from Youssoufia rock phosphate. Fuel., 76:1395-1400.

Khan MS, Zaidi A, Wani PA. 2009. Role of phosphate solubilizing microorganisms in sustainable agriculture. In Sustainable Agriculture, Lichtfouse E, Navarrete M, Debaeke P, Véronique S, Alberola C (eds). Springer; 551-570. DOI: 10.1007/978-90-481-2666-8_34.

Liu D, Coloe S, Baird R, Pedersen J, 2000. Rapid minipreparation of fungal DNA for PCR. J. Clin. Microbiol., 38: 4711471.

Logan NA, Berkeley RC. 1984. Identification of Bacillus strains using the API system. J. Gen. Microbiol., 130: 1871-1882.

Midekssa MJ, Löscher CR, Schmitz RA, Assefa F. 2016. Phosphate solubilization and multiple plant growth promoting properties of rhizobacteria isolated from chickpea (Cicer aeritinum L.) producing areas of Ethiopia. African Journal of Biotechnology, 15(35): 1899-1912. DOI: 10.5897/AJB2015.15172.

Nakamura LK. 1989. Taxonomic relationship of black-pigmented Bacillus subtilis strains and a proposal for Bacillus atrophaeus sp. nov. Int J Syst Bacteriol., 39: 295-300.

Nautyal CS. 1999. An efficient microbiological growth medium for screening of phosphate solubilizing 
microorganisms. FEMS Microbiol Lett, 170: $265-270$.

Nguimbi E, Li YZ, Gao Bl, Li ZF, Wang B, Wu ZH, Yan BX, Qu YB, Gao PJ. 2003. 16S-23S Ribosomal DNA Intergenic Spacer Regions in Cellulolytic Myxobacteria and Differentiation of Closely Related Strains System. Appl. Microbiol., 26: 262-268.

Ouardi E. 2008. Étude de la calcination du phosphate clair de Youssoufia (Maroc). Afrique SCIENCE, 04(02): 199-211.

Ouhdouch Y. 1996. Antibiotiques antifongiques produits par les actinomycètes : : screnning, caractérisation structurale et métabolique d'une souche active de Kitasatosporia. Thèse de Doctorat d'Etat, Université Cadi Ayyad, 218p.

Pikovskaya RI. 1948. Mobilization of phosphorus in soil in connection with vital activity of some microbial species. Microbiology, 17: 362-370.

Popavath RN, Gurusamy R, Kannan BN, Natarajan S. 2008. Assessment of genetic and functional diversity of phosphate solubilizing fluorescent pseudomonads isolated from rhizospheric soil. BMC Microbiology, 8: 230. DOI:10.1186/1471- 2180-8-230.

Pradhan N, Sukla LB. 2005. Solubilization of inorganic phosphate by fungi isolated from agriculture soil. African $J$. Biotechnol., 5(10) : 850-854.

Rivière J. 1975. Les Applications Industrielles de la Microbiologie. Masson et Cie Editeurs : Paris.
Roberts MS, Nakamura LK, Cohan FM. 1994. Bacillus mojavensis sp. nov., distinguishable from Bacillus subtilis by sexual isolation, divergence in DNA sequence, and differences in fatty acid composition. Int. J. Syst. Bacteriol., 44: 256-264.

Ruiz-García C, Béjar V, Martínez-Checa F, Llamas I, Quesada E. 2005. Bacillus velezensis sp. nov., a surfactantproducing bacterium isolated from the river Vélez in Málaga, southern Spain. Int. J. Syst. Evol. Microbiol., 55: 191195.

Stackebrandt E, Liesack W, Webb R, Witt D. 1991. Towards a molecular identification of Streptomyces species in pure culture and environmental samples. Actinomycetes, 2: 54-61.

Whitelaw MA. 2000. Growth promotion of plants inoculated with phosphate solubilizing fungi. Adv. Agron., 69: 99.

Xiao CQ, Chi RA, Li WS, Zheng Y. 2011. Biosolubilization of phosphorus from rock phosphate by moderately thermophilic and mesophilic bacteria. Miner. Eng., 24:956-958.

Zaidi A, Khan M, Ahemad M, Oves M, Wani PA. 2009. Recent Advances in Plant Growth Promotion by PhosphateSolubilizing Microbes. In Microbial Strategies for Crop Improvement, Khan MS, Zaidi A, Musarrat J (eds). Springer: Berlin, Germany; 23-50. 